\title{
On the Application of Cooperative Transmission to Wireless Broadcast Channels
}

\author{
Zhiguo Ding ${ }^{1}$, Kin K. Leung ${ }^{2}$, Dennis L. Goeckel ${ }^{3}$ and Don Towsley ${ }^{4}$ \\ ${ }^{1}$ Department of Communication Systems, Lancaster, UK \\ ${ }^{2}$ Electrical Engineering Department, Imperial College, London, UK \\ ${ }^{3}$ Dept. of Electrical and Computer Engineering, University of Massachusetts, Amherst, MA, US \\ ${ }^{4}$ Dept. of Computer Science, University of Massachusetts, Amherst, MA, US.
}

\begin{abstract}
In this paper, we study the application of cooperative diversity to wireless broadcast channels, a fundamental building block of wireless communication networks. Several cooperative broadcast protocols will be proposed, and information theoretic metrics are developed to facilitate performance evaluation. Provided that there is no direct S-D link, the proposed protocols can achieve a multiplexing gain close to one, whereas the traditional two-hop scheme can only achieve the diversity gain $1 / 2$. Provided that there are direct S-D links, the proposed protocol can still outperform the comparable scheme, particularly at high multiplexing gains.
\end{abstract}

\section{INTRODUCTION}

Cooperative diversity has been extensively studied for the simple scenario of one source-destination pair has been extensively studied (e.g. [1]-[3]), and the application of cooperative diversity to multi-user scenarios has gained increased attention. In this paper we focus on the application of cooperative diversity to the broadcast channel, which is one of the fundamental building blocks of wireless communication networks. The design of cooperative broadcast protocols is quite challenging as the broadcast channel is severely limited by co-channel interference. One approach to avoid such co-channel interference is to assume that there are no individual messages and each destination is to decode all source messages. As shown in [3], such a cooperative broadcast protocol can realize diversity gain larger than one for low multiplexing gains, but no diversity gain can be achieved for multiplexing gains over $1 / 2$. In [4], the capacity regions for relay broadcast channels have been developed; however, the impact of fading and the achievable diversity gain were not addressed.

In this paper, we focus on the application of cooperative diversity to fading broadcast channels, where a source tries to deliver individual messages to destination nodes with the help of relays. In contrast to the broadcast protocol proposed in [3], inactive nodes are used as relays and exploited as an extra dimension for performance improvement. The use of such dedicated relays is motivated by the fact that there are more inactive nodes than active nodes in many practical systems. For example, in a lecture hall, the total number of mobile users is large, and only a few will make phone calls at any particular time. Hence the reminder of the mobile users can be

K. K. Leung, D. L. Goeckel and D. Towsley are was sponsored by US Army Research laboratory and the UK Ministry of Defence and was accomplished under Agreement Number W911NF-06-3-0001, and by the US National Science Foundation under grant CNS-0721861. Z. Ding was supported by the UK EPSRC under grant number EP/F062079/1. exploited as relays and the number of relays can be quite large. Similar observations can be found in other practical systems, such as dense sensor networks. The contributions of this paper are two-fold. First for scenarios without direct S-D links, a new cooperative broadcast protocol is devised that realizes a multiplexing gain of $\frac{M}{M+1}$, where $M$ denotes the number of destinations. Note that a traditional two-hop transmission scheme with relay selection can only achieve the multiplexing gain $1 / 2$ as shown in [2]. In addition, two simple modifications are applied to the proposed broadcast protocol to enhance system performance. One, inspired by cognitive radio, pushes the achievable diversity gain close to the maximum diversity gain. The second is used to increase the multiplexing gain to $\xi$ which approaches one when there are sufficient reliable relays.

Secondly for scenarios with direct S-D links, distributed beamforming is applied to the broadcast channel and a new cooperative transmission protocol is developed to realize a diversity gain of $(L-M+1)$ up to the multiplexing gain $\frac{M}{M+1}$, where $L$ denotes the number of the relays. On the other hand, recall that the cooperative broadcast protocols proposed in [3] cannot achieve diversity gains larger than one when the multiplexing gains is larger than $1 / 2$.

\section{Transmission Strategy for the ScEnario WITHOUT DIRECT S-D LINKS}

Consider a communication scenario with one source, $M$ destinations, and $L$ relays. Each of the $M$ destinations is to receive a different message from the source with the help of relays. Similar to [1], the half-duplex constraint is imposed on the nodes, and time division duplexing is used because of its simplicity. In this section, it is assumed that there is no direct link between the source and destinations, as in [2].

\section{A. Protocol description}

The proposed transmission strategy consists of two phases: initialization and data transmission. During initialization, relay selection is preformed, which ensures that only the relays with good channel conditions will participate in the data transmission. In specific, consider the index set of relays that can decode a rate $\frac{M+1}{M} R$ transmission from the source $\left\{i:\left|h_{S R_{i}}\right|^{2} \geq \frac{2^{\frac{(M+1)}{M} R}-1}{\rho}\right\}$, where $\rho$ denotes signal-to-noise ratio (SNR), $R$ denotes the targeted data rate to each destination, and $h_{S R_{i}}$ denotes the channel between the source and the $i$-th relay. Assume this set contains $K$ relays, $K \leq L$, and 
denote them as $\left\{R_{1}, \ldots, R_{K}\right\}$. It is assumed that $K \geq M$; otherwise, the two-hop relaying scheme proposed in [2] can be employed. Each relay is assumed to have the access to its local CSI.

For data transmissions, each data frame consists of $P=$ $M+1$ time slots as shown in Fig. 1. During the first time slot, the source broadcasts the message $s_{1}$ at the intended rate $\frac{M+1}{M} R$ which is intended for the first destination. All $K$ qualified relays decode and store $s_{1}$. The relay, $R_{1}^{*}$, that has the best connection to the first destination is chosen to forward $s_{1}$ during the next time slot (i.e. $\left|h_{R_{1}^{*} D_{1}}\right|^{2}=$ $\max \left\{\left|h_{R_{1} D_{1}}\right|^{2}, \ldots,\left|h_{R_{K} D_{1}}\right|^{2}\right\}$ ). During the second time slot, the source broadcasts a new message, $s_{2}$, at the intended rate $\frac{M+1}{M} R$ intended for the second destination. At the same time, the chosen relay $R_{1}^{*}$ will forward the message $s_{1}$ to the first destination. Since there are no direct S-D links, the first destination only observes the message transmitted by $R_{1}^{*}$. Although the rest of the $K-1$ qualified relays observe the mixture of $s_{1}$ and $s_{2}$, the qualification criteria guarantees that each of them can decode $s_{2}$ via successive decoding, since they all have decoded and stored $s_{1}$. Due to the half-duplex constraint, the relay $R_{1}^{*}$ will miss the chance to receive the message $s_{2}$, which means this relay cannot be reused in the following time slots, and hence the relay $R_{1}^{*}$ is removed from the qualified relay set for succeeding relaying.

Similarly, at the $m$-th time slot $(2 \leq m \leq(M-1))$, the source broadcasts the message $s_{m}$, and the relay $R_{m-1}^{*}$ forwards the message $s_{m-1}$, where the relay $R_{m-1}^{*}$ is chosen because it has the best connection to the $(m-1)$-th destination among the remaining $(K-m+1)$ qualified relays. At the last time slot, the relay $R_{M}^{*}$ is the only transmitter and it delivers $s_{M}$ to the $M$-th destination.

\section{B. Outage performance and diversity-multiplexing tradeoff}

Outage probability will be used as the criterion for performance evaluation since the probability of error of the maximum likelihood (ML) detector can be tightly bounded by the outage probability at high SNR [5]. Denote $\mathcal{O}$ by the overall outage event for broadcast channel and $\mathcal{O}_{K}$ by the outage event when there are $K$ qualified relays. Then

$$
P(\mathcal{O})=\sum_{k=0}^{L} P\left(\mathcal{O}_{k}\right) P(K=k) .
$$

Recall the definition of the diversity gain and multiplexing gain as [5]

$$
d \triangleq-\lim _{\rho \rightarrow \infty} \frac{\log \left[P_{e}(\rho)\right]}{\log \rho}, \quad \text { and }, \quad r \triangleq \lim _{\rho \rightarrow \infty} \frac{R(\rho)}{\log \rho},
$$

where $P_{e}$ is the ML probability of detection error. Furthermore, $f(\rho)$ is said to be exponentially equal to $\rho^{d}$, denoted as $f(\rho) \doteq \rho^{d}$, when $\lim _{\rho \rightarrow \infty} \frac{\log [f(\rho)]}{\log \rho}=d$. The following theorem provides the achievable diversity-multiplexing tradeoff conditioned on $K$ for the proposed scheme

Theorem 1: Assume all wireless channels are independent identically Rayleigh faded and there are no direct links between the source and destinations. Provided that there are
$K$ qualified relays and $K \geq M$, the overall outage probability can be approximated as

$$
P\left(\mathcal{O}_{K}\right) \doteq \rho^{-d_{t w o_{-} h o p, K}(r)},
$$

where $d_{\text {two_hop, } K}(r)=(K-M+1)\left(1-\frac{M+1}{M} r\right)$, for $0<$ $r<\frac{M}{M+1}$.

Proof: See [6].

In the case $K<M$, the proposed protocol cannot work; hence we define $P\left(\mathcal{O}_{K}\right)=1$ when $K<M$. Note that the number of the qualified relays $K$ is dynamically changing according to instantaneous channel conditions, so it is of interest to relate the achievable diversity-multiplexing tradeoff with the total number of the relays, $L$. The probability for the event that there are $K$ qualified relays is [7]

$$
P(K=k) \doteq \rho^{(L-k)\left(1-\frac{M}{M+1} r\right)},
$$

for $0 \leq k \leq L$. Combining (1), (4) and (3), we can find the achievable diversity-multiplexing tradeoff of the proposed protocol as

$d_{\text {two } h o p}=(L-M+1)\left(1-\frac{M+1}{M} r\right), \quad 0<r<\frac{M}{M+1}$.

Recall that the two-hop transmission scheme with relay selection of [2] achieves the diversity-multiplexing tradeoff

$$
d\left(r_{c}\right)=L(1-2 r),
$$

which means the maximum multiplexing gain achieved is $1 / 2$. However, the proposed transmission protocol can realize a multiplexing gain of $\frac{M}{M+1}$. If the number of destinations is large, the proposed broadcast scheme achieves a multiplexing gain close to one.

Compared to the optimal single-input multiple-output upper bound, the multiplexing and diversity gain achieved by the proposed scheme is less. So in the following, two simple approaches will be applied to the proposed protocol, which is to improve multiplexing and diversity gain.

\section{A cognitive radio inspired approach}

The main reason for the loss in diversity gain is that relays cannot be reused. At the $m$-th time slot, $m-1$ relays have been used previously and hence only $K-m+1$ relays are available for the $(m-1)$-th user. The key step to increase diversity gain is to enable relay reuse.

Take the $m$-th time slot as an example. According to the proposed protocol, two messages will be transmitted simultaneously, $s_{m}$ by the source and $s_{m-1}$ by a relay, $R_{m-1}^{*}$. Denote $\mathcal{R}_{m}$ as the relay which has the best connection to the $m$-th destination, and consider that this relay has been scheduled previously, which means that the relay $\mathcal{R}_{m}$ does not have the priori information about $s_{m-1}$. As a result, the relay $\mathcal{R}_{m}$ is not able to decode $s_{m}$ from the received mixture, and hence is not able to help the $m$-th user.

Interestingly such a scenario can be viewed as a classical cognitive radio problem. The source and $\mathcal{R}_{m}$ are the secondary transmitter and receiver, whereas $R_{m-1}^{*}$ and the destination are the primary transmitter and receiver. It is interesting to observe that the secondary transmitter has the priori information about 
the message transmitted by the primary transmitter [8]. Extensive studies have been carried out for such a problem, where sophisticated design of the secondary transceiver has been proposed to realize perfect interference avoidance, such as dirty paper coding (DPC) and Tomlinson Harashima precoding (THP) [9]. For simplicity, we focus on a simple precoding design of the secondary transmitter. Specifically, we ask the source to transmit the symbol ${ }^{1} s_{m}-\frac{h_{R_{m-1}^{*} \mathcal{R}_{m}}}{h_{S \mathcal{R}_{m}}} s_{m-1}$, instead of $s_{m}$. To realize such a cognitive approach, the inter-relay CSI is required. As a result, the relay $\mathcal{R}_{m}$ receives the mixture of the two messages transmitted by the source and $R_{m-1}^{*}$

$y_{\mathcal{R}_{m}}=h_{S \mathcal{R}_{m}}\left(s_{m}-\frac{h_{R_{m-1}^{*} \mathcal{R}_{m}}}{h_{S \mathcal{R}_{m}}} s_{m-1}\right)+h_{R_{m-1}^{*} \mathcal{R}_{m}} s_{m-1}+n_{\mathcal{R}_{m}}$,

This allows a relay that has already been scheduled to rejoin in the cooperation. However, an important observation of this approach is that a node cannot be scheduled during two consecutive time slots, due to the half duplex constraint. Hence, it still possible that a user cannot get the help from its best relay, but the use of the proposed approach can ensure that at least the second best relay is available. By using such an approach, the overall achievable diversity-multiplexing tradeoff for the modified broadcast protocol is

$$
d_{\text {two_hop }}=(L-1)\left(1-\frac{M+1}{M} r\right), \quad 0<r<\frac{M}{M+1} .
$$

Hence the proposed approach can achieve the diversity gain $L-1$, which is the maximum diversity gain minus one.

\section{A Simple Approach to increase the multiplexing gain}

The main reason for the loss in multiplexing gain is that one extra time slot is required for relay transmission compared with the schemes in [1], [3]. An effective way to increase the multiplexing gain is to reduce the ratio $\frac{1}{P}$ where $P$ is the frame length. In particular, consider $K=q M+p$, where $q=\left\lfloor\frac{K}{M}\right\rfloor$ and $p$ is the residual. Both $q$ and $p$ are integers. For the scheme proposed in the previous section, only $M$ relays out of the $K$ qualified ones were employed. Alternatively, we can use all $K$ relays with the length of the frame set to $(K+1)$. In particular, at the $n$-th time slot $(1<n<(K+1))$, the $[(n$ $\bmod M)-1]$-th destination will be served by a relay and the source broadcasts a new message simultaneously. As a result, $p$ destinations will be served $(q+1)$ times, and the rest $(M-p)$ ones will be served $q$ times. Take a scenario with $K=5$ and $M=2$ as an example. The destinations served from the second time slot to the last time slot are $\left[D_{1}, D_{2}, D_{1}, D_{2}, D_{1}\right]$.

By using such an approach, we can have the following lemma for the achievable diversity-multiplexing tradeoff.

Lemma 2: Provided that there are $K$ qualified relays and $K \geq M$ and the cognitive approach is not applied, the

\footnotetext{
${ }^{1}$ The simple approach outlined requires the relaxation of the transmission power; however, the use of more advanced precoding schemes, such as DPC and THP, can avoid such a problem. Also note that the probability of the case that a desirable relay has previously been used is small given a large number of relays, which means that there are only a few occasions for such a relaxation of the transmission power.
}

achievable outage probabilities for the $m$-th destination can be upper bounded as

$$
P\left(\mathcal{O}_{K}\right) \dot{\leq} \rho^{K-M+1-\frac{K+1}{K}(K+M-p) r},
$$

where $K=q M+p$. Provided that the cognitive approach is applied, the overall achievable outage probability can be upper bounded as

$$
P\left(\mathcal{O}_{K}\right) \dot{\leq} \rho^{(K-1)\left(1-\left(\frac{K+1}{K}\right)^{\frac{1}{q}} r\right)} .
$$

Proof: See [6].

Remarks By using this lemma, it can be easily evaluated that the achievable multiplexing gain with $K$ qualified relays approaches one when $K$ is large. Such a property is particularly important for many applications, such as a cellular network with many mobile users, or a dense sensor network.

\section{TRAnsmission Strategy FOR the SCEnARIO With DIRECT S-D LINKS}

In this section, we consider that the destinations can hear the source directly. Opportunistic scheduling will be carried out, where the $M$ destinations are served in a order reversely proportional to their connections to the source. Or in the other words, the destination with the poorest connection to the source will be scheduled first, and the one with the best connection will be served at last. Without loss of generality, we denote these nodes as $D_{1}, \cdots, D_{M}$, where their connections to the source are ordered as $\left|h_{S D_{1}}\right|^{2} \leq \cdots \leq\left|h_{S D_{M}}\right|^{2}$ and $h_{S D_{1}}$ denotes the channel between the source and $D_{1}$. A distributed strategy of relay selection will be carried out during data transmissions, where we denote $R_{i}$ as the relay selected to transmit during the $(i+1)$-th time slot. The details of relay selection will be provided later.

\section{A. Protocol Description and Signal Model}

During the first time slot, the source broadcasts the message $s(1)$ which is a mixture of the two symbols, $s(1)=\alpha_{11} s_{1}+$ $\alpha_{12} \tilde{s}_{2}$, where $s_{1}$ is the message for the first destination and the structure of $\tilde{s}_{2}$ will be discussed later as well as the design for the weighting factors, $\alpha_{11}$ and $\alpha_{12}$. Each of the $L$ relays receives

$$
y_{R_{i}}(1)=h_{S R_{i}} s(1)+n_{R_{i}}(1),
$$

where $n_{R_{i}(1)}$ is the additive white complex Gaussian noise at the $i$-th relay with power $P_{n}$.

At the second time slot, two nodes will be transmitting simultaneously. The source will transmit a new message, $s(2)=\alpha_{13} s_{1}+\alpha_{14} \tilde{s}_{2}$, and the chosen relay will forward the message $\frac{y_{R_{i}}(1)}{\beta_{R_{1}}}$ where $\beta_{R_{1}}=\sqrt{\left|h_{S R_{1}}\right|^{2}+\frac{1}{\rho}}$ is to normalize the transmission power. As a result, the first destination $D_{1}$ receives

$y_{D_{1}}=\mathbf{h}_{D_{1}}\left[\begin{array}{cc}\alpha_{13} & \alpha_{14} \\ \frac{h_{S R_{1}} \alpha_{11}}{\beta_{R_{1}}} & \frac{h_{S R_{1} \alpha_{12}}}{\beta_{R_{1}}}\end{array}\right]\left[\begin{array}{c}s_{1} \\ \tilde{s}_{2}\end{array}\right]+n_{D_{1}}+\frac{h_{R_{1} D_{1}}}{\beta_{R_{1}}} n_{R_{1}}$.

where $\mathbf{h}_{D_{1}}=\left[\begin{array}{ll}h_{S D_{1}} & h_{R_{1} D_{1}}\end{array}\right]$. To avoid co-channel interference and ensure each destination to observe their own 
messages, the precoding matrix, $\mathbf{P}_{1}$, should be designed to stratify the following requirements

$$
\left[\begin{array}{ll}
\alpha_{13} & \alpha_{14} \\
\alpha_{11} & \alpha_{12}
\end{array}\right]=\left[\begin{array}{cc}
\frac{h_{S D_{1}}^{*}}{\gamma_{11}} & \frac{h_{R_{1} D_{1}}}{\gamma_{12}} \\
\frac{h_{R_{1} D_{1}}^{*} \beta_{R_{1}}}{h_{S R_{1}} \gamma_{12}} & -\frac{h_{S D_{1}} \beta_{R_{1}}}{h_{S R_{1}} \gamma_{12}}
\end{array}\right]
$$

where $\gamma_{11}=\sqrt{\left|h_{S D_{1}}\right|^{2}+\frac{\left|h_{R_{1} D_{1}}\right|^{2} \beta_{R_{1}}^{2}}{\left|h_{S R_{1}}\right|^{2}}}$ and $\gamma_{12}=$ $\sqrt{\left|h_{R_{1} D_{1}}\right|^{2}+\frac{\left|h_{S D_{1}}\right|^{2} \beta_{R_{1}}^{2}}{\left|h_{S R_{1}}\right|^{2}}}$. So at high SNR, we can observe that the two factors $\gamma_{1 i}$ are the same because $\beta_{R_{1}}^{2} \approx$ $\left|h_{S R_{1}}\right|^{2}$. To simplify the notation, we use $\gamma_{1}=\gamma_{1 i}=$ $\sqrt{\left|h_{S D_{1}}\right|^{2}+\left|h_{R_{1} D_{1}}\right|^{2}}$.

By using the proposed precoding matrix, the signal model at the first destination can be simplified as

$$
y_{d_{1}}=\left(\frac{\left|h_{S D_{1}}\right|^{2}}{\gamma_{1}}+\frac{\left|h_{R_{1} D_{1}}\right|^{2}}{\gamma_{1}}\right) s_{D_{1}}+n_{D_{1}}+\frac{h_{R_{1} D_{1}}}{\beta_{R_{1}}} n_{R_{1}},
$$

which demonstrates that co-channel interference has been removed completely. Furthermore, the above signal model shows that the proposed precoding matrix can ensure coherent combining of the messages transmitted from the source and relay. The achievable mutual information for the first destination can be obtained as

$$
\mathcal{I}_{1}=\frac{M}{M+1} \log \left(1+\rho \frac{\left(\left|h_{S D_{1}}\right|^{2}+\left|h_{R_{1} D_{1}}\right|^{2}\right)\left|h_{S R_{1}}\right|^{2}}{\left|h_{R_{1} D_{1}}\right|^{2}+\left|h_{S R_{1}}\right|^{2}}\right) .
$$

On the other hand, the rest relay $R_{i}$ coherently combines its observations over the two time slots and obtains the following

$$
y_{R_{i}}=h_{S R_{i}} \tilde{s}_{2}+\tilde{n}_{R_{i}}
$$

where $\tilde{n}_{R_{i}}$ can be approximated as a white complex Gaussian noise with power $P_{n}$, the same as $n_{R_{1}}$, provided that the number of relays is sufficiently large. The details to obtain (11) can be found in [6]. While $\tilde{n}_{R_{i}}$ is correlated with $h_{S R_{1}}$ and $h_{R_{1} D_{1}}$, such a correlation will not cause any implications to the signal model of other nodes during the following time slots due to the assumption that all addressed channels are i.i.d Rayleigh faded.

Following similar steps, the $m$-th destination will be served at the $(m+1)$-th time slot, and its achievable data rate can be expressed as

$\mathcal{I}_{m}=\frac{M}{M+1} \log \left(1+\rho \frac{\left(\left|h_{S D_{m}}\right|^{2}+\left|h_{R_{m} D_{m}}\right|^{2}\right)\left|h_{S R_{m}}\right|^{2}}{\left|h_{R_{m} D_{m}}\right|^{2}+\left|h_{S R_{m}}\right|^{2}}\right)$,

for $1 \leq m \leq M-1$. The only exception happens at the $(M+1)$-th time slot, where the source sends out the message $\frac{h_{S D_{M}}^{*}}{\theta} s_{M}$ and the relay $R_{M}$ forwards the message $\frac{h_{R_{M} D_{M}}^{*}}{\theta}\left(s_{M}+\frac{\tilde{n}_{R_{M}}}{h_{S R_{M}}}\right)$, where $\theta^{2}=\left|h_{S D_{M}}\right|^{2}+\left|h_{R_{M} D_{M}}\right|^{2}+$ $\frac{1}{\mid h_{\left.S R_{M}\right|^{2} \rho}}$. It can be easily verified that the expression in (12) is a lower bound for the data rate realized at the $M$-th user.

Relay selection can be implemented in a distributed way as in [10]. An important question is how to design a criterion to measure channel conditions. Studying the expression of the supportable data rate shown in (12), we can conclude that the optimal criterion for relay selection should be

$$
\frac{\left(\left|h_{S D_{1}}\right|^{2}+\left|h_{R_{1} D_{1}}\right|^{2}\right)\left|h_{S R_{1}}\right|^{2}}{\left|h_{R_{1} D_{1}}\right|^{2}+\left|h_{S R_{1}}\right|^{2}} .
$$

However, such a criterion requires that each relay knows nonlocal CSI, $h_{S D_{m}}$, which could consume extra system overhead. Instead the following expression will be used for the criterion of relay selection

$$
\frac{\left|h_{R_{1} D_{1}}\right|^{2}\left|h_{S R_{1}}\right|^{2}}{\left|h_{R_{1} D_{1}}\right|^{2}+\left|h_{S R_{1}}\right|^{2}}
$$

which is the Harmonic mean of the two channel channels $\left|h_{R_{1} D_{1}}\right|^{2}$ and $\left|h_{S R_{1}}\right|^{2}$. The benefit of the criterion in (14) is that each relay only needs to have the access to its local CSI.

The following theorem provides the outage performance achieved by the proposed protocol.

Theorem 3: Assume all addressed wireless channels are i.i.d. Rayleigh faded and the destinations can hear the source directly. The outage probability achieved by the proposed cooperative broadcast protocol can be approximated at high SNR as

$$
P(\mathcal{O}) \doteq \rho^{-d(r)},
$$

where $d(r)=(L-M+1)\left(1-\frac{M+1}{M} r\right)$, for $0<r<\frac{M}{M+1}$. Proof: See [6].

Recall that the amplified-forward broadcast protocol proposed in [3] can achieve the tradeoff as

$$
d\left(r_{c}\right)=(1-r)+(N-1)(1-2 r)^{+},
$$

which means that diversity gain no larger than one can be achieved for the multiplexing gain $\frac{1}{2} \leq r \leq 1$. However, Theorem 3 demonstrates that the diversity gain larger than one can be achieved by the proposed transmission protocol for the multiplex gain up to $\frac{M}{M+1}$. Provided that the number of the destination nodes is large enough, we can have $\frac{M}{M+1} \rightarrow 1$.

While increasing the number of the destinations increases the multiplexing gain, it decreases the achievable diversity gain. Again we can apply the simple approach to increase the multiplexing gain proposed in Section II-D. As a result, even with a small $M$, the diversity-multiplexing tradeoff can be approximated as $d(r) \approx(L-M+1)(1-r)$.

\section{NumERICAL RESUlTS}

When there is no direct S-D link, the comparable protocol is the traditional two-hop transmission scheme with relay selection [2]. In specific, $2 M$ time slots are required in total, where each destination is allocated two consecutive time slots and its serving relay is chosen as the best one among the $L$ relays. To simplify notations in the figures, we denote the protocols proposed in Section II-A and Section II-D as Type I and II respectively. In addition, we denote the combination of the approaches in Section II-C and II-D as Type III.

In Fig.2, the outage probability is shown as a function of SNR, where the number of the relays and destinations is $L=6$ and $M=3$, and the targeted data rate is $R=4$ bits per channel use (BPCU). As can be seen from the figure, the proposed protocols can achieve better outage performance 
than the classical two-hop scheme with relay selection. The reason for such a performance gain is due to the fact that the proposed protocols can achieve the multiplexing gain at least $\frac{M}{M+1}$, whereas the comparable scheme can only achieve the multiplexing gain up to $1 / 2$. Furthermore, the two figures demonstrate that the use of the simple approaches proposed in Section II-C and II-D can enhance the performance of the proposed protocol. In specific, the use of the method discussed in Section II-D can improve the achievable multiplexing gain when $K>M$, and the use of the cognitive approach can increase the diversity gain from $L-M+1$ to $L-1$.

When there are direct S-D links, the comparable schemes are the non-cooperative direct transmission scheme and the non-orthogonal amplify forward (NAF) based protocol [3].The number of the relays and destination nodes is $L=4$ and $M=2$. The targeted data rate is $R=3$ BPCU. Again we denote the protocol proposed in Section III-A as the proposed CB protocol Type I, and its enhanced version by serving destination nodes more than one times as Type II. Furthermore, the performance for the proposed protocols with two different relay selection criteria, the optimal one in (13) and the suboptimal one in (14), has also been shown. As can be observed from Fig. 3, the proposed broadcast transmission protocols can outperform the comparable schemes. The reason for such a performance gain is due to the fact that the NAF scheme cannot provide a diversity gain larger than one for the multiplexing gain $\frac{1}{2} \leq r \leq 1$, whereas the use of the proposed protocols can yield the diversity gain $(L-M+1)$ up to the multiplexing gain $\frac{M}{M+1}$.

\section{CONClusion}

In this paper, several new cooperative transmission protocols for wireless broadcast channels have been described. The dynamic nature of fading channels has been utilized to improve the performance of cooperative networks. Both analytical and numerical results have been provided to demonstrate that the proposed protocols can achieve a better performance than comparable schemes.

\section{REFERENCES}

[1] J. N. Laneman, D. N. C. Tse, and G. W. Wornell, "Cooperative diversity in wireless networks: Efficient protocols and outage behavior," IEEE Trans. Information Theory, vol. 50, pp. 3062-3080, Dec. 2004.

[2] R. U. Nabar, H. Bolcskei, and F. W. Kneubuhler, "Fading relay channels : performance limits and space-time signal design," IEEE Journal on Select. Areas in Comm., vol. 22, pp. 1099-1109, Aug. 2004.

[3] K. Azarian, H. E. Gamal, and P. Schniter, "On the achievable diversitymultiplexing tradeoff in half-duplex cooperative channels," IEEE Trans. Information Theory, vol. 51, pp. 4152-4172, Dec. 2005.

[4] Y. Liang and G. Kramer, "Rate regions for relay broadcast channels," IEEE Trans. Information Theory, vol. 50, pp. 3517-3535, Feb. 2007.

[5] D. N. C. Tse, P. Viswanath, and L. Zheng, "Diversity-multiplexing tradeoff in multiple-access channels," IEEE Trans. Information Theory, vol. 50, pp. 1859-1874, Sept. 2004.

[6] Z. Ding, K. K. Leung, D. L. Goeckel, and D. Towsley, "Cooperative transmission protocols for wireless broadcast channels," IEEE Trans. on Communications, (submitted).

[7] Z. Ding, Y. Gong, T. Ratnarajah, and C. Cowan, "On the performance of opportunistic cooperative wireless networks," IEEE Trans. on Communications, vol. 56, pp. 1236-1240, Aug. 2008.

[8] N. Devroye, P. Mitran, and V. Tarokh, "Achievable rates in cognitive radio channels," IEEE Trans. Information Theory, vol. 52, pp. 18131827, May 2006.

[9] C. C. Kuo, C. C. J. Kuo, S.-H. Tsai, L. Tadjpour, and Y.-H. Chang, Precoding Techniques for Digital Communication Systems. Springer Press, 2008.

[10] A. Bletsas, A. Khisti, D. P. Reed, and A. Lippman, "A simple cooperative diversity method based on network path selection," IEEE Journal on Select. Areas in Comm., vol. 24, pp. 659-672, Mar. 2006.

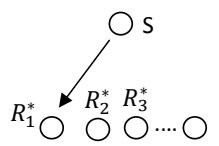

$\bigcirc \bigcirc \bigcirc \cdots \bigcirc$

$\begin{array}{llll}D_{1} & D_{2} & D_{3} & D_{M}\end{array}$
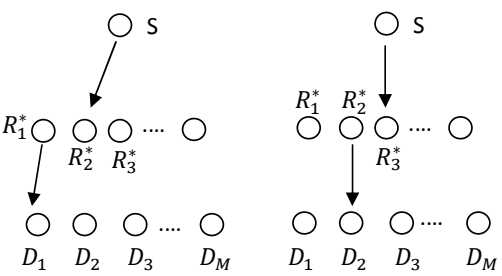

Fig. 1. A diagram for the description of the proposed broadcast transmission protocol.

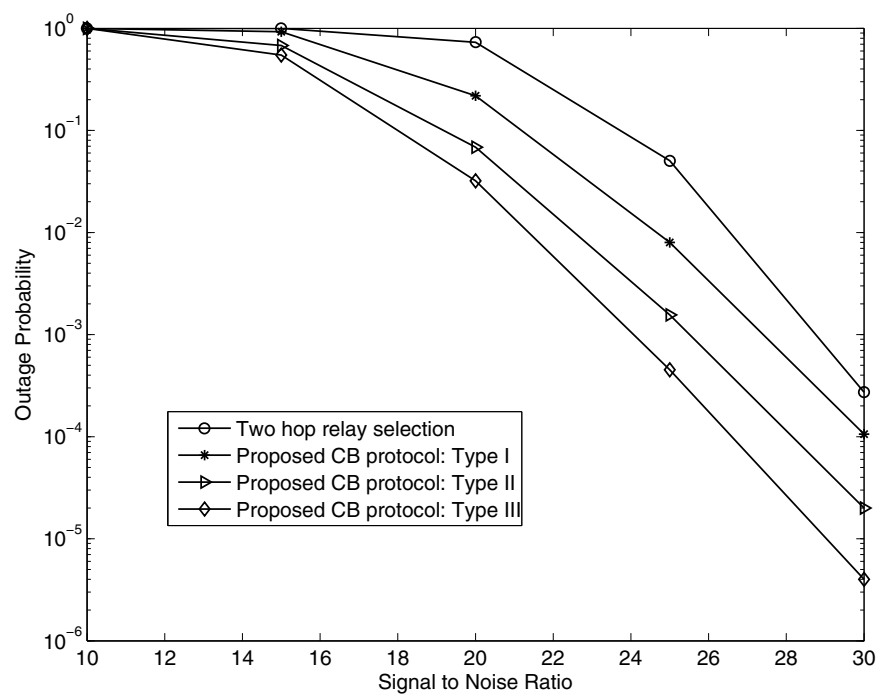

Fig. 2. The outage probability achieved by the proposed broadcast transmission protocols and the comparable schemes vs SNR.

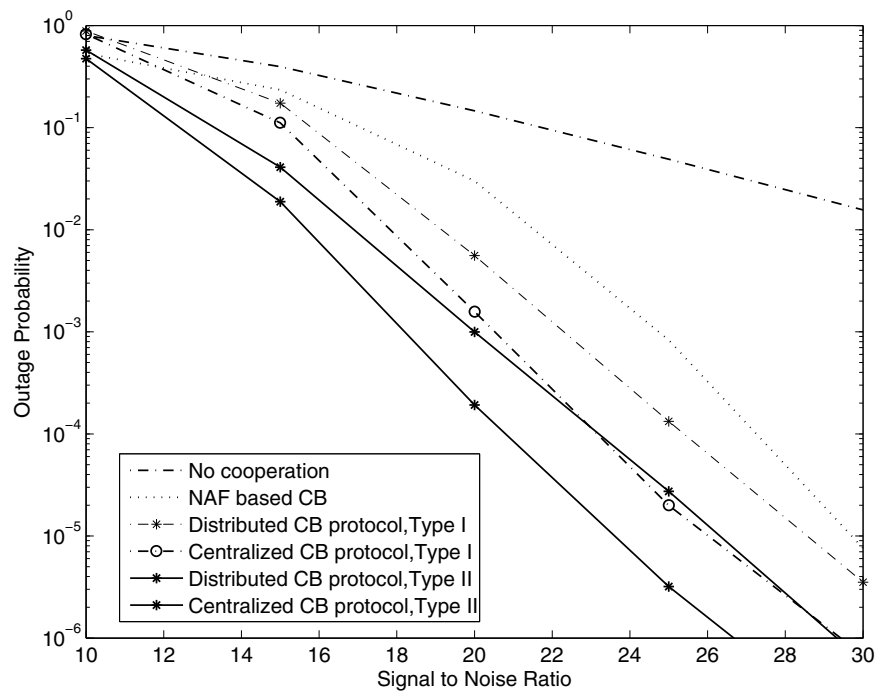

Fig. 3. The outage probability achieved by the proposed broadcast transmission protocols and the comparable schemes vs SNR. 